\title{
Hyperspectral Signal Subspace Estimation
}

\author{
José M. P. Nascimento \\ Instituto Superior de Engenharia de Lisboa \\ and Instituto de Telecomunicações \\ R. Conselheiro Emídio Navarro, \\ N. 1, edifício DEETC, \\ 1959-007 Lisboa, Portugal \\ Tel.:+351.21.8317237, Fax:+351.21.8317114, \\ Email: zen@isel.pt
}

\author{
José M. Bioucas-Dias \\ Instituto de Telecomunicações \\ Instituto Superior Técnico \\ Technical University of Lisbon \\ Av. Rovisco Pais, Torre Norte, Piso 10 \\ 1049-001 Lisboa, Portugal \\ Tel.: +351.21.8418466, Fax:+351.21.8418472, \\ Email: bioucas@1x.it.pt
}

\begin{abstract}
Given an hyperspectral image, the determination of the number of endmembers and the subspace where they live without any prior knowledge is crucial to the success of hyperspectral image analysis.

This paper introduces a new minimum mean squared error based approach to infer the signal subspace in hyperspectral imagery. The method, termed hyperspectral signal identification by minimum error (HySime), is eigendecomposition based and it does not depend on any tuning parameters. It first estimates the signal and noise correlation matrices and then selects the subset of eigenvalues that best represents the signal subspace in the least squared error sense. The effectiveness of the proposed method is illustrated using simulated data based on U.S.G.S. laboratory spectra and real hyperspectral data collected by the AVIRIS sensor over Cuprite, Nevada.
\end{abstract}

\section{INTRODUCTION}

Hyperspectral imaging sensors collect two dimensional spatial images from the Earth's surface over many contiguous bands of high spectral resolution covering the visible, nearinfrared, and shortwave infrared (wavelengths between $0.3 \mu \mathrm{m}$ and $2.5 \mu \mathrm{m}$ ), in hundreds of narrow (on the order of $10 \mathrm{~nm}$ ) contiguous spectral bands. For example, AVIRIS collects a 512 (along track) $\times 614$ (across track) $\times 224$ (bands) $\times 12$ (bits) data cube, corresponding to more than 800 Mbits [1]. Such huge data volumes put stringent requirements in what concerns communications, storage, and processing.

Each pixel of an hyperspectral image can be represented as a vector in the Euclidian space $\mathbb{R}^{L}$, where $L$ is the number of bands and each channel is assigned to one axis of space. Under the linear mixing scenario, the spectral vectors are a linear combination of the so-called endmember signatures. The number of endmembers present in a given scene is, very often, much less than the number of bands $L$. Therefore, hyperspectral vectors lie in a low dimensional linear subspace. The identification of this subspace enables the representation spectral vectors in a low dimensional subspace, thus yielding gains in computational time and complexity and in data storage.

Principal component analysis (PCA) and maximum noise fraction (MNF) are two techniques often used to reduce the dimensionality of hyperspectral data. The first technique seeks the projection that best represents data in the least square sense, whereas the second seeks the projection that optimizes the ratio of noise power to signal power. In addition, MNF method needs to estimate the noise covariance. Minimum description length (MDL) [2,3] and Akaike information criterion (AIC) [4] have also been used to infer the hyperspectral signal subspace.

Harsanyi, Farrand, and Chang [5] developed a NeymanPearson detection theory-based thresholding method (HFC) to determine the number of spectral endmembers in hyperspectral data, referred to in [6] as virtual dimensionality (VD). The HFC method uses the eigenvalues to measure signal energies in the detection model. A modified version, termed noisewhitened HFC (NWHFC), includes a noise-whitening step [6].

This paper proposes a new minimum mean squared error based approach to determine the signal subspace in hyperspectral imagery. The method, termed hyperspectral signal identification by minimum error (HySime), starts by estimating the signal and the noise correlation matrices using multiple regression. The eigenvectors of the signal correlation matrix are then used to build a sequence of nested subspaces. The signal subspace is inferred by minimizing the sum of the projection error power with the noise power, which are, respectively, decreasing and increasing functions of the subspace dimension. Therefore, if the subspace dimension is overestimated the noise power term is dominant, whereas if the subspace dimension is underestimated the projection error power term is the dominant. The overall scheme is adaptive in the sense that it does not depend on any tuning parameters. Paper [7] further details and extends the ideas herein presented.

Section II describes the fundamentals of the HySime method. Sections III and IV evaluate the proposed algorithm using simulated and real data, respectively. Section V ends the paper by presenting a some concluding remarks.

\section{HySime Method Description}

This section introduces formally the HySime algorithm. The method starts by estimating the signal and the noise correlation matrices and then it selects the subset of eigenvectors that best represents the signal subspace in the minimum mean squared error sense. The application of this criterium leads to the minimization of a two-term objective function. One term 
corresponds to the power of the signal projection error and is a decreasing function of the subspace dimension; the other term corresponds to the power of the noise projection and is an increasing function of subspace dimension.

Let us assume an hyperspectral image where each pixel $\mathbf{y}$ can be represented as a spectral vector in $\mathbb{R}^{L}$ ( $L$ is the number of bands), i.e.,

$$
\mathbf{y}=\mathbf{x}+\mathbf{n},
$$

where $\mathbf{x}$ and $\mathbf{n}$ are $L$-dimensional vectors standing for signal and additive noise, respectively.

\section{A. Noise Estimation}

Noise estimation is a classical problem in data analysis and particulary in remote sensing. Multiple regression theory based approaches have been applied to hyperspectral noise estimation $[6,8]$. These approaches outperform the shift difference method, as they exploit the high correlation existing among hyperspectral bands.

Let us denote by $\mathbf{Y}$ an $L \times N$ matrix holding the $N$ spectral observed vectors of size $L$. Define the matrix $\mathbf{Z}=\mathbf{Y}^{T}$, the $N \times 1$ vector $\mathbf{z}_{i}=[\mathbf{Z}]_{:, i}$, where $[\mathbf{Z}]_{:, i}$ stands for the $i$ th column of $\mathbf{Z}$ (i.e., $\mathbf{z}_{i}$ contains the data read by the hyperspectral sensor at the $i$ th band for all image pixels), and the $N \times(L-1)$ matrix $\mathbf{z}_{\partial_{i}}=\left[\mathbf{z}_{1}, \ldots, \mathbf{z}_{i-1}, \mathbf{z}_{i+1}, \ldots, \mathbf{z}_{L}\right]$.

Assume that $\mathbf{z}_{i}$ is explained by a linear combination of the remaining $L-1$ bands. Formally, this consists in writing

$$
\mathbf{z}_{i}=\mathbf{Z}_{\partial_{i}} \beta_{i}+\xi_{i},
$$

where $\mathbf{Z}_{\partial_{i}}$ is the explanatory data matrix, $\beta_{i}$ is the regression vector of size $(L-1) \times 1$, and $\xi_{i}$ is the modeling error vector of size $N \times 1$. For each $i \in\{1, \ldots, L\}$, the least squares estimator of the regression vector is given by $\widehat{\beta}_{i}=\left(\mathbf{Z}_{\partial_{i}}^{T} \mathbf{Z}_{\partial_{i}}\right)^{-1} \mathbf{Z}_{\partial_{i}}^{T} \mathbf{z}_{i}$, the noise is estimated by $\widehat{\xi}_{i}=\mathbf{z}_{i}-\mathbf{Z}_{\partial_{i}} \widehat{\beta}_{i}$, and the correlation matrix by $\widehat{\mathbf{R}}_{n}=\left[\widehat{\xi}_{1}, \ldots, \widehat{\xi}_{N}\right]^{T}\left[\widehat{\xi}_{1}, \ldots, \widehat{\xi}_{N}\right] / N$. Notice that the determination of each noise vector $\widehat{\xi}_{i}$ implies the computation of the pseudo-inverse $\mathbf{Z}_{\partial_{i}}^{\#}=\left(\mathbf{Z}_{\partial_{i}}^{T} \mathbf{Z}_{\partial_{i}}\right)^{-1} \mathbf{Z}_{\partial_{i}}^{T}$, of size $(L-1) \times(L-1)$, for each $i=1, \ldots, L$. This huge computational complexity can, however, be greatly reduced by taking advantage of the relation between $\mathbf{Z}_{\partial}^{\#}$ and $\mathbf{Z}$ (see details in [7]). For $N \gg L$, the algorithm implemented in [7] demands, approximately, $L / 2$ less flops (number of float point operations), what is a significant figure since $L / 2$ takes, in many applications, values on the order of 100 .

\section{B. Signal Subspace Estimation}

This section presents the core structure of the proposed method. The first step, based on the noise estimation procedure introduced in the previous section, identifies a set of orthogonal directions of which an unknown subset spans the signal subspace. This subset is then determined by seeking the minimum mean squared error between $\mathbf{x}$, the original signal, and a noisy projection of it obtained from the vector $\mathbf{y}=\mathbf{x}+\mathbf{n}$.
In the following, we assume that $\mathbf{n} \sim \mathcal{N}\left(\mathbf{0}, \widehat{\mathbf{R}}_{n}\right)$, i.e., the noise is zero-mean Gaussian distributed with covariance ma$\operatorname{trix} \widehat{\mathbf{R}}_{n}$.

Let the eigen-decomposition of the signal sample correlation matrix $\widehat{\mathbf{R}}_{x}=\frac{1}{N} \sum_{i}\left(\left(\mathbf{y}_{i}-\widehat{\xi}_{i}\right)\left(\mathbf{y}_{i}-\widehat{\xi}_{i}\right)^{T}\right)$ be written as

$$
\widehat{\mathbf{R}}_{x}=\mathbf{E} \Sigma \mathbf{E}^{T},
$$

where $\mathbf{E} \equiv\left[\mathbf{e}_{1}, \ldots, \mathbf{e}_{k}, \mathbf{e}_{k+1} \ldots, \mathbf{e}_{L}\right]$ is a matrix with the eigenvectors, $\mathbf{e}_{j}$, ordered by the decreasing magnitude of the respective eigenvalues. The space $\mathbb{R}^{L}$ can be decomposed into two orthogonal subspaces: $\left\langle\mathbf{E}_{k}\right\rangle$ spanned by $\mathbf{E}_{k} \equiv\left[\mathbf{e}_{1}, \ldots, \mathbf{e}_{k}\right]$ and $\left\langle\mathbf{E}_{k}\right\rangle^{\perp}$ spanned by $\mathbf{E}_{k}^{\perp} \equiv\left[\mathbf{e}_{k+1}, \ldots, \mathbf{e}_{L}\right]$, i.e., the orthogonal complement of subspace $\mathbf{E}_{k}$, where $k$ is the order of the signal subspace.

Let $\mathbf{U}_{k}=\mathbf{E}_{k} \mathbf{E}_{k}^{T}$ be the projection matrix onto $\left\langle\mathbf{E}_{k}\right\rangle$ and $\widehat{\mathbf{x}}_{k} \equiv$ $\mathbf{U}_{k} \mathbf{y}$ be the projection of the observed spectral vector $\mathbf{y}$ onto the subspace $\left\langle\mathbf{E}_{k}\right\rangle$. The first and the second-order moments of $\widehat{\mathbf{x}}_{k}$ given $\mathbf{x}$ are

$$
\begin{aligned}
\mathbb{E}\left[\widehat{\mathbf{x}}_{k} \mid \mathbf{x}\right] & =\mathbf{U}_{k} \mathbb{E}[\mathbf{y} \mid \mathbf{x}] \\
& =\mathbf{U}_{k} \mathbb{E}[\mathbf{x}+\mathbf{n} \mid \mathbf{x}] \\
& =\mathbf{U}_{k} \mathbf{x} \\
& \equiv \mathbf{x}_{k}, \\
\mathbb{E}\left[\left(\widehat{\mathbf{x}}_{k}-\mathbf{x}_{k}\right)\left(\widehat{\mathbf{x}}_{k}-\mathbf{x}_{k}\right)^{T} \mid \mathbf{x}\right] & =\mathbb{E}\left[\left(\mathbf{U}_{k} \mathbf{n} \mathbf{n}^{T} \mathbf{U}_{k}^{T}\right) \mid \mathbf{x}\right] \\
& =\mathbf{U}_{k} \widehat{\mathbf{R}}_{n} \mathbf{U}_{k}^{T} .
\end{aligned}
$$

The mean squared error between $\mathbf{x}$ and $\widehat{\mathbf{x}}_{k}$ is

$$
\begin{aligned}
\operatorname{mse}(k \mid \mathbf{x}) & =\mathbb{E}\left[\left(\mathbf{x}-\widehat{\mathbf{x}}_{k}\right)^{T}\left(\mathbf{x}-\widehat{\mathbf{x}}_{k}\right) \mid \mathbf{x}\right] \\
& =\mathbb{E}\left[\left(\mathbf{x}-\mathbf{x}_{k}-\mathbf{U}_{\mathbf{k}} \mathbf{n}\right)^{T}\left(\mathbf{x}-\mathbf{x}_{k}-\mathbf{U}_{\mathbf{k}} \mathbf{n}\right) \mid \mathbf{x}\right] \\
& =\mathbf{b}_{k}^{T} \mathbf{b}_{k}+\operatorname{tr}\left(\mathbf{U}_{k} \widehat{\mathbf{R}}_{n} \mathbf{U}_{k}^{T}\right) .
\end{aligned}
$$

Computing the mean of (6) with respect to $\mathbf{x}$, noting that $\mathbf{b}_{k}=\mathbf{x}-\mathbf{x}_{\mathbf{k}}=\mathbf{U}_{\mathbf{k}}^{\perp} \mathbf{x}$, and using the properties $\mathbf{U}=\mathbf{U}^{T}, \mathbf{U}^{2}=\mathbf{U}$, and $\mathbf{U}^{\perp}=\mathbf{I}-\mathbf{U}$ of the projection matrices, we get

$$
\begin{aligned}
\operatorname{mse}(k) & =\mathbb{E}\left[\left(\mathbf{U}_{k}^{\perp} \mathbf{x}\right)^{T}\left(\mathbf{U}_{k}^{\perp} \mathbf{x}\right)\right]+\operatorname{tr}\left(\mathbf{U}_{k} \widehat{\mathbf{R}}_{n} \mathbf{U}_{k}^{T}\right) \\
& =\operatorname{tr}\left(\mathbf{U}_{k}^{\perp} \mathbf{R}_{x}\right)+\operatorname{tr}\left(\mathbf{U}_{k} \widehat{\mathbf{R}}_{n}\right) \\
& =\operatorname{tr}\left(\mathbf{U}_{k}^{\perp} \mathbf{R}_{y}\right)+2 \operatorname{tr}\left(\mathbf{U}_{k} \widehat{\mathbf{R}}_{n}\right)+c,
\end{aligned}
$$

where $c$ is an irrelevant constant. The criteria we propose to estimate the signal subspace is the minimization of $\operatorname{mse}(k)$ given by (7) with the correlation matrix $\mathbf{R}_{y}$ replaced with the sample correlation matrix $\widehat{\mathbf{R}}_{y}=\mathbf{Y} \mathbf{Y}^{T} / N$, i.e.,

$$
\widehat{k}=\arg \min _{k}\left\{\operatorname{tr}\left(\mathbf{U}_{k}^{\perp} \widehat{\mathbf{R}}_{y}\right)+2 \operatorname{tr}\left(\mathbf{U}_{k} \widehat{\mathbf{R}}_{n}\right)\right\},
$$

Each term of expression (8) has a clear meaning: the first accounts for the projection error power and is a decreasing function of $k$; the second accounts for the noise power and is an increasing function of $k$. 


\section{Evaluation of HySime with Simulated Data}

In this section, we apply the proposed HySime algorithm to simulated scenes and compare it with the NWHFC eigenbased Neyman-Pearson detector [6]. As concluded in [6], these algorithms are the state-of-the-art in hyperspectral signal subspace identification, outperforming the information theoretical criteria approaches; namely, the minimum description length (MDL) [2, 3] and the Akaike information criterion (AIC) [4].

A simulated hyperspectral image composed of $10^{4}$ spectral vectors, each one following the linear mixing model (1), is generated. Assume that signal vectors are in an $p$-dimensional subspace, i.e.,

$$
\mathbf{x}=\mathbf{M s},
$$

where $\mathbf{M} \equiv\left[\mathbf{m}_{1}, \mathbf{m}_{2}, \ldots, \mathbf{m}_{p}\right]$ is a full-rank $L \times p$ matrix $\left(\mathbf{m}_{i}\right.$ denotes the $i$ th endmember signature), $p$ is the number of endmembers present in the covered area, and $\mathbf{s}=\left[s_{1}, s_{2}, \ldots, s_{p}\right]^{T}$ is the abundance vector containing the fractions of each endmember (notation $(\cdot)^{T}$ stands for vector transposed).

The spectral signatures are selected from the USGS digital spectral library [9]. The abundance fractions are generated according to a Dirichlet distribution given by

$$
D\left(s_{1}, \ldots, s_{p} \mid \theta_{1}, \ldots, \theta_{p}\right)=\frac{\Gamma\left(\sum_{j=1}^{p} \theta_{j}\right)}{\prod_{j=1}^{p} \Gamma\left(\theta_{j}\right)} \prod_{j=1}^{p} s_{j}^{\theta_{j}-1},
$$

where $\left\{s_{1}, \ldots, s_{p}\right\}$ are subject to nonnegativity and constant sum constraints, i.e., $\left\{\mathbf{s} \in \mathbb{R}^{p}: s_{j} \geq 0, \sum_{j=1}^{p} s_{j}=1\right\}$. This density, besides enforcing positivity and full additivity constraints, displays a wide range of shapes, depending on the parameters of the distribution. On the other hand, as noted in [10], the Dirichlet density is suited to model fractions.

Consider that the noise correlation matrix is $\mathbf{R}_{n}=$ $\operatorname{diag}\left(\sigma_{1}^{2}, \ldots, \sigma_{L}^{2}\right)$ and that the diagonal elements follow a Gaussian shape centered at the band $L / 2$, i.e.,

$$
\sigma_{i}^{2}=\sigma^{2} \frac{e^{-\frac{(i-L / 2)^{2}}{\left(2 \eta^{2}\right)}}}{\sum_{j=1}^{L} e^{-\frac{(j-L / 2)^{2}}{\left(2 \eta^{2}\right)}}},
$$

for $i=1, \ldots, L$. Parameter $\eta$ plays the role of variance in the Gaussian shape $(\eta \rightarrow \infty$ corresponds to white noise; $\eta \rightarrow$ 0 corresponds to one-band noise). Parameter $\sigma^{2}$ controls the total noise power.

The method is evaluated with respect to the number of endmembers $p$, to the spectral noise shape (white and nonwhite), and to the SNR defined by

$$
\mathrm{SNR} \equiv 10 \log _{10} \frac{\mathbb{E}\left[\mathbf{x}^{T} \mathbf{x}\right]}{\mathbb{E}\left[\mathbf{n}^{T} \mathbf{n}\right]}
$$

Fig. 1 shows the evolution of the mean squared error for HySime algorithm as a function of the parameter $k$, for $p=5, \mathrm{SNR}=20 \mathrm{~dB}$ and $\eta=1 / 18$. The minimum of the mean squared error occurs at $k=5$, which is exactly the number of endmembers present in the image. As expected, the projection error power and of noise power display decreasing

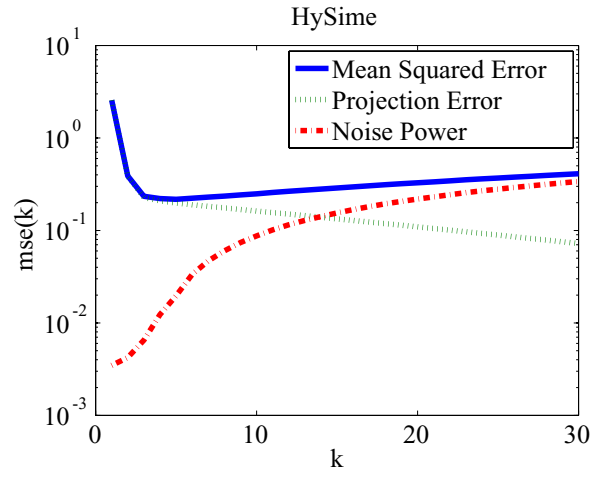

Fig. 1: Mean squared error versus $k$, with $S N R=20 \mathrm{~dB}, p=5$

and increasing behaviors, respectively, as a function of the subspace dimension $k$.

Table I presents the signal subspace order estimates yielded by HySime algorithm and the virtual dimensionality (VD) determined by the NWHFC algorithm [6], as a function of the SNR, of the number of endmembers, $p$, and of the noise shape.

NWHFC algorithm is basically the HFC one [5] preceded by a noise-whitening step, based on the estimated noise correlation matrix. In implementing this step, we got poor results in very high SNRs and colored noise scenarios. For this reason, we have used both the true and estimated noise correlation matrices. The results based on the true correlation matrix are in brackets. We stress that, for the setting of this experiment, HySime method yields the same results, whether using the estimated or the true noise correlation matrices.

Another central issue of NWHFC algorithm is the falsealarm probability $P_{f}$ it is parameterized with. This probability is used in a series of Neyman-Pearson tests, each one designed to detect a different orthogonal signal subspace direction. It is necessary, therefore, to specify the false-alarm probability $P_{f}$ of the tests. Based on the hints given in [6] and in our own results, we choose $P_{f} \in\left\{10^{-3}, 10^{-4}, 10^{-5}\right\}$.

The figures shown in Table I, based on 50 Monte Carlo runs, have the following behavior:

i) HySime and NWHFC algorithms parameterized with $P_{f}=10^{-3}$ display similar performances at low subspace dimension, say $p \leq 5$, and white noise. This is also true for colored noise and NWHFC working with known noise covariance matrix. However, if the noise statistics is unknown, NWHFC performs much worse than HySime;

ii) HySime performs better that NWHFC for high space dimensions, say $p>5$.

We conclude, therefore, that HySime algorithm yields systematically equal or better results than NWHFC algorithm. Another advantage of HySime approach is that it does not depend on any tunable parameter.

\section{EXPERIMENTS WITH REAL HyPERSPECTRAL DATA}

In this section, the proposed method, HySime, is applied to a subimage $(350 \times 350$ pixels and 224 bands $)$ of the Cuprite 
TABLE I: Signal subspace dimension $\widehat{k}$, based on 50 Monte Carlo runs, as function of SNR, $p$, and $\eta$ (noise shape). Figures in brackets were computed based on the true noise statistics.

\begin{tabular}{|c|c|c|c|c|c|}
\hline & & \multicolumn{4}{|c|}{ White Noise $(\eta=0)$} \\
\hline SNR & Method & $p=3$ & $p=5$ & $p=10$ & $p=15$ \\
\hline \multirow{4}{*}{$50 \mathrm{~dB}$} & HySime & 3 & 5 & 10 & 15 \\
\hline & $\operatorname{NWHFC}\left(P_{f}=10^{-3}\right)$ & $3(3)$ & $5(5)$ & $7(7)$ & $10(11)$ \\
\hline & $\operatorname{NWHFC}\left(P_{f}=10^{-4}\right)$ & $3(3)$ & $5(5)$ & $7(7)$ & $8(8)$ \\
\hline & NWHFC $\left(P_{f}=10^{-5}\right)$ & $3(3)$ & $4(4)$ & $7(6)$ & $8(8)$ \\
\hline \multirow{4}{*}{$35 \mathrm{~dB}$} & HySime & 3 & 5 & 10 & 15 \\
\hline & $\operatorname{NWHFC}\left(P_{f}=10^{-3}\right)$ & $3(3)$ & $4(4)$ & $7(7)$ & $9(9)$ \\
\hline & $\operatorname{NWHFC}\left(P_{f}=10^{-4}\right)$ & $3(3)$ & $4(4)$ & $7(6)$ & $8(8)$ \\
\hline & $\operatorname{NWHFC}\left(P_{f}=10^{-5}\right)$ & $3(3)$ & $4(4)$ & $6(6)$ & $8(8)$ \\
\hline \multirow{4}{*}{$25 \mathrm{~dB}$} & HySime & 3 & 5 & 10 & 14 \\
\hline & $\operatorname{NWHFC}\left(P_{f}=10^{-3}\right)$ & $3(3)$ & $5(5)$ & $6(6)$ & $9(8)$ \\
\hline & $\operatorname{NWHFC}\left(P_{f}=10^{-4}\right)$ & $3(3)$ & $5(5)$ & $6(6)$ & 7 (7) \\
\hline & $\operatorname{NWHFC}\left(P_{f}=10^{-5}\right)$ & $3(3)$ & $4(4)$ & $5(5)$ & $7(7)$ \\
\hline \multirow{4}{*}{$15 \mathrm{~dB}$} & HySime & 3 & 5 & 8 & 12 \\
\hline & $\operatorname{NWHFC}\left(P_{f}=10^{-3}\right)$ & $3(3)$ & $5(5)$ & $5(4)$ & $5(5)$ \\
\hline & $\operatorname{NWHFC}\left(P_{f}=10^{-4}\right)$ & $3(3)$ & $4(4)$ & $3(3)$ & $3(2)$ \\
\hline & $\operatorname{NWHFC}\left(P_{f}=10^{-5}\right)$ & $3(3)$ & $4(4)$ & $3(3)$ & $2(2)$ \\
\hline \multirow[b]{2}{*}{ SNR } & & \multicolumn{4}{|c|}{ Gaussian shaped noise $(\eta=1 / 18)$} \\
\hline & Method & $p=3$ & $p=5$ & $p=10$ & $p=15$ \\
\hline \multirow{4}{*}{$50 \mathrm{~dB}$} & HySime & 3 & 5 & 10 & 15 \\
\hline & $\operatorname{NWHFC}\left(P_{f}=10^{-3}\right)$ & $59(3)$ & $41(5)$ & $61(10)$ & $45(10)$ \\
\hline & $\operatorname{NWHFC}\left(P_{f}=10^{-4}\right)$ & 48 (3) & $33(5)$ & $54(10)$ & $34(10)$ \\
\hline & $\operatorname{NWHFC}\left(P_{f}=10^{-5}\right)$ & $43(3)$ & $28(5)$ & $41(9)$ & $27(10)$ \\
\hline \multirow{4}{*}{$35 \mathrm{~dB}$} & HySime & 3 & 5 & 10 & 15 \\
\hline & $\operatorname{NWHFC}\left(P_{f}=10^{-3}\right)$ & $9(3)$ & $10(5)$ & $12(10)$ & $10(10)$ \\
\hline & $\operatorname{NWHFC}\left(P_{f}=10^{-4}\right)$ & $9(3)$ & $9(5)$ & $11(10)$ & $8(10)$ \\
\hline & $\operatorname{NWHFC}\left(P_{f}=10^{-5}\right)$ & $7(3)$ & $7(5)$ & $10(9)$ & $8(10)$ \\
\hline \multirow{4}{*}{$25 \mathrm{~dB}$} & HySime & 3 & 5 & 10 & 15 \\
\hline & $\operatorname{NWHFC}\left(P_{f}=10^{-3}\right)$ & $4(3)$ & $5(5)$ & $11(10)$ & $9(11)$ \\
\hline & $\operatorname{NWHFC}\left(P_{f}=10^{-4}\right)$ & $4(3)$ & $5(5)$ & $11(10)$ & $9(10)$ \\
\hline & $\operatorname{NWHFC}\left(P_{f}=10^{-5}\right)$ & $4(3)$ & $5(5)$ & $11(9)$ & $8(10)$ \\
\hline \multirow{4}{*}{$15 \mathrm{~dB}$} & HySime & 3 & 5 & 8 & 12 \\
\hline & $\operatorname{NWHFC}\left(P_{f}=10^{-3}\right)$ & $4(3)$ & $5(5)$ & $11(10)$ & $10(10)$ \\
\hline & $\operatorname{NWHFC}\left(P_{f}=10^{-4}\right)$ & $4(3)$ & $5(5)$ & $11(10)$ & $8(10)$ \\
\hline & $\operatorname{NWHFC}\left(P_{f}=10^{-5}\right)$ & $4(3)$ & $5(5)$ & $11(9)$ & $8(10)$ \\
\hline
\end{tabular}

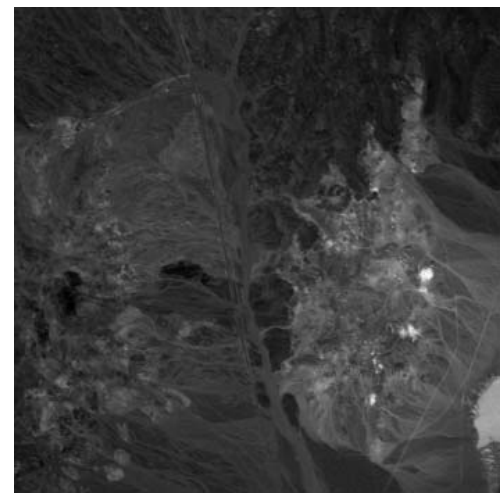

Fig. 2: Band 30 (wavelength $\lambda=655.8 \mathrm{~nm}$ ) of the subimage of AVIRIS Cuprite Nevada data set;

data set acquired by the AVIRIS sensor on June 19, 1997 (see Fig. 2). The AVIRIS instrument covers the spectral region from $0.41 \mu \mathrm{m}$ to $2.45 \mu \mathrm{m}$ in 224 bands with a $10 \mathrm{~nm}$ band width. Flying at an altitude of $20 \mathrm{~km}$, it has an IFOV of $20 \mathrm{~m}$ and views a swath over $10 \mathrm{~km}$ wide. This site has been extensively used for remote sensing experiments over the past years and its geology was previously mapped in detail [11]. The HySime method when applied to the AVIRIS data set, estimates a subspace dimension of $\widehat{k}=20$. According to the ground truth presented in [11], there are 18 materials in this area. This difference is due to a) the presence of rare pixels not accounted for in [11] and b) spectral variability.

The VD estimated by the NWHFC method [6] $\left(P_{f}=10^{-3}\right)$ on the same data set yields $\widehat{k}=23$. A lower value of $P_{f}$ would lead to a higher number of endmembers. According to the ground truth presented in [11], the estimates yielded by HySime and NWHFC methods overestimate the number of endmembers in the Cuprite data set. The mainly reason for this difference, as we have explained, is the presence of rare pixels present in the data set not accounted for in [11].

\section{COnClusions}

The huge volumes and rates of data generated by hyperspectral sensors demand expensive processors with very high performance and memory capacities. Dimensionality reduction is, therefore, a relevant first step in the hyperspectral data processing chain. This paper introduces the HySime algorithm, a new approach to estimate the signal subspace in hyperspectral imagery. HySime algorithm estimates the signal and the noise correlation matrices and then selects the subset of eigenvalues that best represents the signal subspace in the minimum mean squared error sense. A set of experiments with simulated and real data leads to the conclusion that the HySime algorithm is an effective and useful tool, yielding comparable or better results than the state-of-the-art algorithms.

\section{ACKNOWLEDGEMENTS}

This work was supported by the FCT, under the projects POSC/EEA-CPS/61271/2004 and PDCTE/CPS/49967/2003 and by IPL under project IPL-5828/2004.

\section{REFERENCES}

[1] J. P. Kerekes and J. E. Baum, "Spectral imaging system analytical model for subpixel object detection," IEEE Trans. Geosci. Remote Sensing, vol. 40, no. 5, pp. 1088-1101, 2002.

[2] G. Schwarz, "Estimating the dimension of a model," Annals of Statistics, vol. 6, pp. 461-464, 1978.

[3] J. Rissanen, "Modeling by shortest data description," Automatica, vol. 14 , pp. $465-471,1978$.

[4] H. Akaike, "A new look at the statistical model identification," IEEE Trans. Automat. Contr., vol. 19, no. 6, pp. 716-723, 1974.

[5] J. Harsanyi, W. Farrand, and C.-I. Chang, "Determining the number and identity of spectral endmembers: An integrated approach using neyman-pearson eigenthresholding and iterative constrained rms error minimization," in Proc. 9th Thematic Conf. Geologic Remote Sensing, 1993.

[6] C.-I. Chang and Q. Du, "Estimation of number of spectrally distinct signal sources in hyperspectral imagery," IEEE Trans. Geosci. Remote Sensing, vol. 42, no. 3, pp. 608-619, 2004.

[7] J. M. Bioucas-Dias and J. M. P. Nascimento, "Hyperspectral subspace identification," IEEE Trans. Geosci. Remote Sensing, 2007, submmited.

[8] R. Roger and J. Arnold, "Reliably estimating the noise in aviris hyperspectral imagers," Int. J. Rem. Sens., vol. 17, no. 10, pp. 19511962, 1996.

[9] R. N. Clark, G. A. Swayze, A. Gallagher, T. V. King, and W. M. Calvin, "The U.S. geological survey digital spectral library: Version 1: 0.2 to 3.0 m," U.S. Geological Survey, Open File Report 93-592, 1993.

[10] T. Minka, "Estimating a dirichlet distribution," M.I.T., Tech. Rep., 2000.

[11] G. Swayze, R. Clark, S. Sutley, and A. Gallagher, "Ground-truthing aviris mineral mapping at cuprite, nevada," in Summaries of the Third Annual JPL Airborne Geosciences Workshop, 1992, pp. 47-49. 